\title{
Early Isomerization of Bilirubin in Phototherapy of Neonatal Jaundice
}

\author{
KHALAF MREIHIL, ANTONY F. MCDONAGH, BRITT NAKSTAD, AND THOR WILLY RUUD HANSEN
}

\begin{abstract}
Neonatal Intensive Care Unit [K.M., B.N.], Children's and Youth Clinic, Akershus University Hospital, N-1478 Nordbyhagen, Norway; Department of Neonatal Intensive Care [K.M., T.W.R.H.], Women's and Children's Clinic, Oslo University Hospital-Rikshospitalet, N-0027 Oslo, Norway; Division of Gastroenterology [A.F.M.], University of California Medical Center, San Francisco, CA 94131; Institute of Clinical Medicine (B.N., T.W.R.H.), Faculty of Medicine, University of Oslo, N-0318 Oslo, Norway
\end{abstract}

\begin{abstract}
Neonatal jaundice is usually treated with phototherapy that converts bilirubin to more polar stereoisomers. These should theoretically be less able to cross the blood-brain barrier. The rates of photoisomer formation and concentrations accumulating in the circulation may have a bearing on the risk of kernicterus. The purpose of this study was to determine the rate of appearance of the major $4 Z, 15 E$ photoisomer of bilirubin during the early stages of phototherapy. Twenty jaundiced neonates were treated with phototherapy, and blood samples were drawn before and at $\sim 15,30,60$, and 120 $\min$ (10 infants) or at $\approx 15,60,120$, and $240 \mathrm{~min}$ (10 infants) after beginning phototherapy. Blood samples were analyzed for total serum bilirubin (TSB) and the $4 Z, 15 E$ photoisomer of bilirubin. Significant $(p<0.0001)$ formation of the $4 Z, 15 E$ photoisomer was detectable within $15 \mathrm{~min}$. The change in TSB from time 0 was insignificant at $120 \mathrm{~min}$ but reached significance at $240 \mathrm{~min}(p<$ 0.001 ). The $4 Z, 15 E$ bilirubin constituted up to $20-25 \%$ of TSB at $2 \mathrm{~h}$ and may not have peaked by $4 \mathrm{~h}$. Further studies are needed to determine whether this early shift in balance between bilirubin isomers with different polarities may impact the risk of bilirubin encephalopathy even before TSB starts to fall. (Pediatr Res 67: 656-659, 2010)
\end{abstract}

$\mathrm{N}$ eonatal jaundice has the potential to cause both acute and chronic derangements in brain function (1-3). It is this potential for toxicity that motivates therapeutic intervention, most commonly phototherapy. Permanent and devastating brain damage due to kernicterus is rare but continues to occur even in industrialized countries (4). Whether more expedient and aggressive therapy might avoid or at least mitigate the damage in such cases is unclear.

Phototherapy causes several structural changes in the bilirubin molecule. The resulting stereoisomers are more polar than the predominant parent IX $\alpha(4 Z, 15 Z)$ isomer, which needs conjugation to be excreted (5). Photoisomers can be detected in serum and in varying degrees in bile and urine during phototherapy, and their more facile elimination compared with the natural form of the pigment is believed to

Received October 27 2009; accepted March 4, 2010.

Correspondence: Thor Willy Ruud Hansen, M.D., Ph.D., Department of Neonatal Intensive Care, Women's and Children's Clinic, Oslo University Hospital-Rikshospitalet, N-0027 Oslo, Norway; e-mail: t.w.r.hansen@medisin.uio.no

Supported by grants from Anne Margrete Bakkes Fond and Nyfødtfondet, Division of Paediatrics, Oslo University Hospital-Rikshospitalet and by intramural funds from the Faculty of Medicine, University of Oslo, Norway. account in part for the therapeutic effect of phototherapy in terms of lowering total serum bilirubin (TSB) levels.

The most rapidly formed photoisomer is one in which one of the bridging double bonds in bilirubin has undergone a cis-trans isomerization from a so-called $Z$ (zusammen) configuration to an $E$ (entgegen) configuration, thereby converting the normal biosynthetic $4 Z, 15 Z$ form of bilirubin to the $4 Z, 15 E$ isomer (5). The two other possible configurational isomers (lumirubin and $4 E, 15 Z$ ) isomer are also formed rapidly, but to a lesser extent. The photochemical reactions that generate the configurational photoisomers occur in femto seconds or faster.

Several authors have studied the rate of appearance of photoisomers in serum during phototherapy $(6,7)$. However, it is difficult to estimate the initial rate of appearance of photoisomers from those studies either because of the presence of significant amounts of photoisomers (12-18\%) in samples taken even before phototherapy was begun or because early time points were not analyzed. It is unclear whether the photoisomers present in the initial samples were produced by exposure of patients to ambient light (8) or by accidental light exposure during collection and processing of the samples. The purpose of this study was to determine the rate of appearance of the major $4 Z, 15 E$ photoisomer of bilirubin during the early stages of standard phototherapy as practiced in Norway.

\section{METHODS}

Design, subjects, and enrollment. The study was observational and took place in the NICUs of Oslo University Hospital, Rikshospitalet, Oslo, and Akershus University Hospital, Nordbyhagen, Norway. Twenty infants who were due to receive phototherapy according to the Norwegian national guidelines (http://www.legeforeningen.no/asset/39055/1/39055_1.pdf) for such treatment were included. None of the infants had received phototherapy before enrollment. The parents of all infants gave written, informed consent to participation. The study was approved by the Norwegian Data Directorate and by the Health Region South-East Committee on Research Ethics, who imposed restrictions on the number of blood samples from each infant that could be collected and analyzed. The patients had a gestational age of $34 \mathrm{wk}$ and $1 \mathrm{~d} \pm 0 \mathrm{wk}$ and $6 \mathrm{~d}$ (mean $\pm \mathrm{SD}$ ), range $27 \mathrm{wk}$ and $6 \mathrm{~d}$ to $41 \mathrm{wk}$ and $0 \mathrm{~d}$, and a birthweight of $2298 \pm 243 \mathrm{~g}$, range $900-5060$. The age at start of phototherapy was $72 \pm 81 \mathrm{~h}($ mean $\pm \mathrm{SD}$; range $18-309 \mathrm{~h}) .{ }^{1}$

Phototherapy setup. Phototherapy was provided by BiliCompact (Weyer $\mathrm{GmbH}$, Kürten-Herweg, Germany) fluorescent units, which contain ten 9-W, 12.7-cm length fluorescent bulbs (BAM/PL9/52, Ralutec 9W/71 G23). According to the manufacturer's spectra, these bulbs have broad Gaussian

Abbreviation: TSB, total serum bilirubin 
emission roughly from $\sim 400-525 \mathrm{~nm}$ with a peak at $450 \mathrm{~nm}$ and intense mercury emission lines at 405,436 , and $546 \mathrm{~nm}$. The radiant power over the range $400-550 \mathrm{~nm}$ is given as $2.3 \mathrm{~W}$ and their "bilirubin-effective radiation intensity" as $20 \mathrm{~W} / \mathrm{m}^{2}$ at a distance of $25 \mathrm{~cm}$.

The units were positioned at a distance of $20-30 \mathrm{~cm}$ from the infants whenever possible. However, for premature infants in closed incubators, the distance was necessarily greater, up to $35-40 \mathrm{~cm}$. Spectral power (irradiance $\times$ size of irradiated area) was enhanced by covering the bed/bassinet inside with white linen and by hanging white linen as curtains around the unit using specially adapted, locally made racks. Infants were treated naked, except for the use of eye protection. Whenever diapers were needed, these were reduced to the minimum practicable size. Irradiance was measured on the babies' top surface and in the flanks using the Pocket $\mathrm{E}_{\mathrm{bi}}$ photometer (LMT Lichtmesstechnik; GmbH, Berlin, Germany). For comparison, a sample setup was also measured with the AirShields PR III Phototherapy Radiometer.

Sample collection and preparation. Samples were drawn for determination of TSB and $4 Z, 15 E$ bilirubin at $0,15,30,60$, and $120 \mathrm{~min}$ for the first 10 patients and at $0,15,60,120$, and 240 min for the next 10 patients. Nursery lights were dimmed according to Newborn Individualized Developmental Care and Assessment Program (NIDCAP) guidelines. Care was taken to work as much as possible in subdued light during blood collection, which typically took 1-2 min. Thereafter, blood samples were kept in dark containers at all times during transport and storage, and preparation by centrifugation and pipetting was performed under red or orange safe-lights.

Analysis of TSB and bilirubin isomers. TSB was measured by cooximetry on OMNI S/cobas b221 blood gas machines (Roche Diagnostics, F. Hoffmann-La Roche Ltd, Basel, Switzerland) immediately after collection. Serum was kept at $-70^{\circ} \mathrm{C}$ and transported on dry ice for subsequent analysis of bilirubin photoisomers by HPLC (9).

The column used for HPLC was a Beckman-Altex Ultrasphere-IP 5- $\mu \mathrm{m}$ C-18 ODS column $(25 \mathrm{~cm} \times 0.46 \mathrm{~cm})$ maintained at $\sim 37^{\circ} \mathrm{C}$ and fitted with a similarly packed precolumn $(4.5 \mathrm{~cm} \times 0.46 \mathrm{~cm})$, and the eluant was $0.1 \mathrm{M}$ di- $n$-octylamine acetate in methanol containing $5 \%(\mathrm{vol} / \mathrm{vol})$ water at a flow rate of $0.75 \mathrm{~mL} / \mathrm{min}$. Eluted peaks were detected with an Agilent multiwavelength diode array detector at $450 \mathrm{~nm}$ (close to the absorbance maxima for $4 Z, 15 Z$ and $4 Z, 15 E$ bilirubins in the eluting solvent), and peak areas, measured by integration using HP ChemStation software, were corrected for differences in the absorbancies of photoisomers at $450 \mathrm{~nm}$ (9). Each frozen serum sample was thawed to $4^{\circ} \mathrm{C}$, and a $20-\mu \mathrm{L}$ aliquot was immediately diluted into $80-\mu \mathrm{L} 0.1-\mathrm{M}$ di- $n$-octylamine acetate in methanol, vortexed and microfuged rapidly, and the supernate was injected onto the column via a $20-\mu \mathrm{L}$ loop. The proportion of $4 Z, 15 E$ bilirubin, relative to $4 Z, 15 Z$ bilirubin, was determined by comparison of peak areas. Relative, to the $4 Z, 15 E$ peak, the peak areas of other photoisomers were very small and less accurately measurable. Because the focus of the study was the most rapidly formed isomer, data on the concentrations of other isomers were not included.

Statistics. Statistical analyses were done by $t$ tests, Tukey's multiple comparison test, and ANOVA using GraphPad Prism, (GraphPad Software, Inc., LA Jolla, CA).

\section{RESULTS}

Irradiance values on top surface and flanks, as well as TSB at the beginning and end of the study periods are shown in Table 1. Comparison measurements with the Air-Shields PR

Table 1. Irradiation and TSB values in patients treated with phototherapy

\begin{tabular}{lccc}
\hline & Mean $\pm \mathrm{SD}$ & Range & $p$ \\
\hline Irradiance* on top surface $\left(\mathrm{W} / \mathrm{m}^{2}\right)$ & $26.7 \pm 7.8$ & $15.7-45.1$ & $\mathrm{ND}$ \\
Irradiance in flanks $\left(\mathrm{W} / \mathrm{m}^{2}\right)$ & $17.1 \pm 9.4$ & $9.4-33.2$ & $\mathrm{ND}$ \\
TSB $(\mu \mathrm{mol} / \mathrm{L})$ at start in patients & $240 \pm 93$ & $125-422$ & $\mathrm{NS} \dagger$ \\
$0-10$ & & & \\
TSB $(\mu \mathrm{mol} / \mathrm{L})$ at end 120 min in & $230 \pm 86$ & $108-396$ & \\
$\quad$ patients $0-10$ & & & \\
TSB $(\mu \mathrm{mol} / \mathrm{L})$ at start in patients & $204 \pm 58$ & $125-316$ & $<0.001 \ddagger$ \\
$\quad 11-20$ & & & \\
$\begin{array}{l}\text { TSB }(\mu \mathrm{mol} / \mathrm{L}) \text { at end } 240 \text { min in } \\
\text { patients } 11-20\end{array}$ & $177 \pm 54$ & $108-272$ & \\
\hline
\end{tabular}

* Measured with a Pocket $\mathrm{E}_{\mathrm{bi}}$ photometer (wavelength interval 380-780 nm).

$\dagger$ Paired, two-tailed $t$ test vs $120 \mathrm{~min}$.

$\$$ Paired, two-tailed $t$ test vs 240 min.

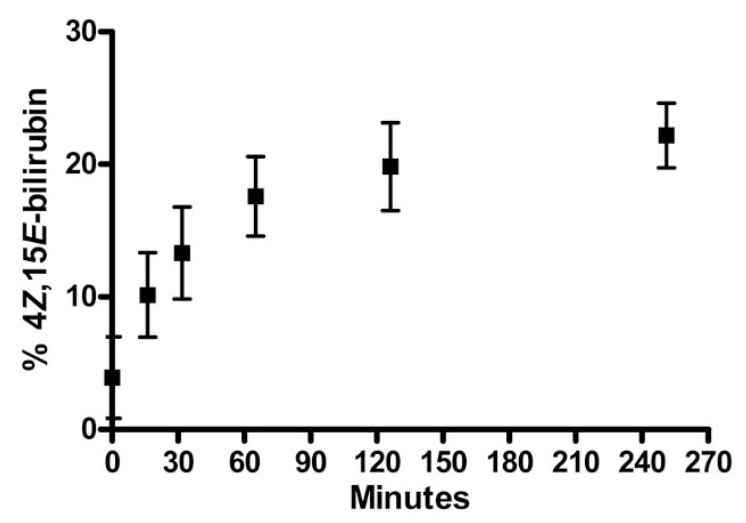

Figure 1. Formation of $4 Z, 15 E$ bilirubin as a percentage (mean \pm SD) of TSB $(4 Z, 15 E+4 Z, 15 Z)$ in 20 jaundiced infants treated with phototherapy $(F$ 79.06, $p<0.0001$, one-way ANOVA). The difference from time 0 was significant from $15 \mathrm{~min}$ onward.

III Phototherapy Radiometer in sample setups yielded irradiance values in $\mu \mathrm{W} / \mathrm{cm}^{2} / \mathrm{nm}$, which were $5-10 \%$ higher in numerical values than the $\mathrm{W} / \mathrm{m}^{2}$ values measured by the Pocket $\mathrm{E}_{\mathrm{bi}}$ photometer. However, as the irradiance in actual patient setups in preliminary studies frequently exceeded the $36 \mu \mathrm{W} / \mathrm{cm}^{2} / \mathrm{nm}$ upper range of the Air-Shields PR III, this measuring device was not used for the actual study, and the relationship between the numbers measured by the two devices may not hold true above the upper range of the AirShields PR III.

In the first group of 10 patients, the change in TSB values from start to end of the brief study period (0-120 min) was not statistically significant when contrasted with a paired, twotailed $t$ test. In the second group of patients, the reduction in TSB from 0-240 min was small but statistically significant (Table 1). The total duration of phototherapy (study period plus continued phototherapy as indicated by clinical guidelines) for all infants was between 7 and $45 \mathrm{~h}$.

Formation of $4 Z, 15 E$ bilirubin was highly significant ( $F$ 79.06, $p<0.0 .0001$, one-way ANOVA), and at all time points starting from $16 \mathrm{~min}$, the percentage of this isomer was significantly higher than at time $0(p<0.05$, Tukey's multiple comparison test; Fig. 1. As seen from the graph, the actual sample collection time points were slightly later than the target times because of the time involved in sample collection.

\section{DISCUSSION}

This study has shown that $4 Z, 15 E$ bilirubin, which begins to form as soon as an infant is exposed to phototherapy lights, is detectable in blood within $15 \mathrm{~min}$. By this time, a mean of $\sim 10 \%$ of circulating bilirubin had undergone isomerization to $4 Z, 15 E$ bilirubin. Small amounts of other photoisomers were also detectable by HPLC but were not quantitated for this study.

In three patients, the proportion of $4 Z, 15 E$ bilirubin reached $14-15 \%$ of the total bilirubin after only 15 min of phototherapy. Interestingly, in two of them, irradiance values $>40$ $\mathrm{W} / \mathrm{m}^{2}$ were measured on the body surface closest to the lights, whereas values $>30 \mathrm{~W} / \mathrm{m}^{2}$ were measured in their flanks. In 
the third patient, corresponding irradiance values were 30.2 and $18.6 \mathrm{~W} / \mathrm{m}^{2}$, respectively. All three had high TSB values when phototherapy was started $(315,254$, and $315 \mu \mathrm{M}$, respectively), and all three were relatively low birthweight, weighing 2670, 1760, and $1680 \mathrm{~g}$, respectively.

Despite precautions to minimize exposure of samples to light, small amounts of $4 Z, 15 E$ bilirubin were present in blood even before phototherapy, although in much smaller amounts than in previous studies $(6,7)$. Whether these isomers hail from the patient, or were the result of accidental ambient light exposure of blood samples during collection and preparation, cannot be answered from our study. The $4 Z, 15 E$ bilirubin has been demonstrated in nonjaundiced subjects exposed to daylight (8). Therefore, it may not be unreasonable to speculate that some photoisomer production will occur in jaundiced infants exposed to bright nursery lights. In recent years, pursuant to the introduction of NIDCAP (10) or similar care principles in many NICUs, ambient nursery lights are likely to be much more subdued than when earlier phototherapy studies were performed (11).

The 2004 American Academy of Pediatrics (AAP) guidelines on management of neonatal jaundice (12) arbitrarily defined intensive phototherapy as an irradiance $>30 \mu \mathrm{W} /$ $\mathrm{cm}^{2} / \mathrm{nm}$ over the wavelength interval $430-490 \mathrm{~nm}$. The mean irradiance value measured on the infants' uppermost surface (i.e. closest to the lights) in this study was $26.7 \pm 7.8 \mathrm{~W} / \mathrm{m}^{2}$ (wavelength interval 380-780 nm). In sample measurements with an older Air-Shields PR III Phototherapy Radiometer that yields values in $\mu \mathrm{W} / \mathrm{cm}^{2} / \mathrm{nm}$ (wavelength interval $330-570$ $\mathrm{nm}$ ), the mean irradiance values in our study seem to be close to the range defined by the AAP as "intensive" despite the low wattage $(9 \mathrm{~W})$ and small size $(\approx 13 \mathrm{~cm})$ of the bulbs used in our units. However, as irradiance measurements may vary between devices depending on the calibration and type of irradiance meter, a meaningful correlation between our $\mathrm{W} / \mathrm{m}^{2}$ measurements and the AAP definition is not possible.

In some previous studies, "360" phototherapy was used, achieved either through specially constructed tube-shaped phototherapy units with cribs that had a translucent bottom or with the infant lying on a fiberoptic mat while receiving fluorescent phototherapy from above and sides (13-15). Initial rates of photoisomer formation with these units are not well defined. Interestingly, using what were described as "special blue" bulbs (TL20/52), Myara et al. (7) observed conversion of $>30 \%$ of the total bilirubin in the serum to Z/E photoisomers in neonates after $3 \mathrm{~h}$ of phototherapy, whereas, using 40-W "violet-blue" (Philips TL40/03) fluorescent tubes, Agati et al. (16) observed $\sim 25 \%$ of conversion within $6 \mathrm{~h}$ in an adolescent Crigler-Najjar type I patient. However, in both of those studies, the proportion of bilirubin photoisomers at time 0 was high (12-18\%). The rate of photoisomerization is a function of the wavelength output of the lights, the intensity of the lights, the surface area of the baby exposed, and possibly the initial serum bilirubin level, whereas the percentage conversion of bilirubin to $4 Z, 15 E$ bilirubin is expected to increase with time to a photostationary value that is dependent only on the wavelengths emitted by the light.
The strength of this study is that the group of patients studied is representative of typical NICU populations regarding the distribution of birth weights and gestational ages. Because of the characteristics of Norwegian guidelines for therapy (which are similar to the AAP guidelines), some infants were treated at relatively low TSB levels. The phototherapy units we have used are commercially available and widely used. The use of white linen inside the crib and hung around the phototherapy unit to increase irradiance by reflection can be replicated anywhere using materials easily available and may increase the efficacy of phototherapy $(17,18)$. Thus, the rates of bilirubin stereoisomer formation that we have found in this study are likely to be achievable in other NICUs as well.

It is noteworthy that after $2 \mathrm{~h}$ of phototherapy, the reduction of TSB levels from time 0 was not significant. This may seem somewhat surprising, given the substantial reductions in TSB levels that have previously been documented within the same time interval (19). However, in that study, the TSB levels were extreme, which predicts a more dramatic response to phototherapy. Significantly, $\sim 20 \%$ of TSB at $2 \mathrm{~h}$ was in the form of the $4 Z, 15 E$ photoisomer. If, as appears possible considering their physicochemical characteristics, photoisomers of bilirubin have less ability to cross the blood-brain barrier than the predominant IX $\alpha(Z, Z)$ isomer, this may translate into a lesser threat to the brain.

Kernicterus regrettably continues to occur, although with due precautions it ought to be avoidable. Reports of infants admitted with extreme jaundice and neurologic symptoms compatible with intermediate to advanced stage acute bilirubin encephalopathy keep surfacing among neonatologists. Recent publications suggest that intermediate to advanced stage acute bilirubin encephalopathy can occasionally be reversible in neonates $(4,20,21)$, as also observed in adult patients with Crigler-Najjar syndrome (22). Expedient and aggressive treatment seems to be a common theme in these reports.

None of the patients in this study had any signs of bilirubininduced neurologic dysfunction. Therefore, reversibility of neurotoxicity during the study period could not be assessed. Analysis of bilirubin in CSF might have answered the question of whether bilirubin efflux from the CNS is dependent on the relative concentrations of $(Z, E)$ vs $(Z, Z)$ bilirubin IX $\alpha$ in serum. However, extant Norwegian ethics rules would not have permitted repeated lumbar punctures for this purpose or is it likely that consent could have been obtained.

Herein, we have confirmed that formation of significant amounts of $4 Z, 15 E$ bilirubin occurs in phototherapy long before any alternative or adjunctive treatment could have been instituted. The clinical implications of converting such a large proportion of a major metabolite in the circulation to a different form, with a different three-dimensional structure, markedly different lipophilicity, and different serum albumin binding properties, are unknown. With respect to bilirubin toxicity, there are, a priori, three possibilities: the photoisomer has similar toxicity to the "natural" isomer; the photoisomer is more toxic; or the photoisomer is less toxic. Of these, the first is unlikely because of the different structures and physicochemical properties of the two forms and the need for pigment 
to enter the brain to cause toxicity. The second possibility also seems unlikely because countless phototherapy treatments over the last half-century seem never to have caused or exacerbated CNS toxicity. This leaves the third possibility; the most likely in view of the much lower lipophilicity of the photoisomer compared with the $4 Z, 15 Z$ isomer, which would be expected to make it less prone to cross the blood-brain barrier and enter the brain. However, the magnitude of such an effect could be insignificantly small, and it remains unknown whether photoisomerization engenders a rapid detoxification process, as proposed long ago (23), or has little effect on the potential toxicity of all forms of bilirubin in the circulation.

\section{REFERENCES}

1. Byers RK, Paine RS, Crothers B 1955 Extrapyramidal cerebral palsy with hearing loss following erythroblastosis. Pediatrics 15:248-254

2. Nwaesei CG, Van Aerde J, Boyden M, Perlman M 1984 Changes in auditory brainstem responses in hyperbilirubinemic infants before and after exchange transfusion. Pediatrics 74:800-803

3. Volpe JJ 2008 Neurology of the Newborn. 5th ed. Elsevier, Philadelphia, PA, pp $1-1042$

4. Johnson L, Bhutani VK, Karp K, Sivieri EM, Shapiro SM 2009 Clinical report from the pilot USA Kernicterus Registry (1992 to 2004). J Perinatol 29 (suppl 1):S25-S45

5. Maisels MJ, McDonagh AF 2008 Phototherapy for neonatal jaundice. N Engl J Med 358:920-928

6. Onishi S, Isobe K, Itoh S, Manabe M, Sasaki K, Fukuzaki R, Yamakawa T 1986 Metabolism of bilirubin and its photoisomers in newborn infants during phototherapy. J Biochem 100:789-795

7. Myara A, Sender A, Valette V, Rostoker C, Paumier D, Capoulade C, Loridon F, Bouillie J, Milliez J, Brossard Y, Trivin F 1997 Early changes in cutaneous bilirubin and serum bilirubin isomers during intensive phototherapy of jaundiced neonates with blue and green light. Biol Neonate 71:75-82

8. McDonagh AF 1986 Sunlight-induced mutation of bilirubin in a long-distance runner. N Engl J Med 314:121-122
9. McDonagh AF, Agati G, Fusi F, Pratesi R 1989 Quantum yields for laser photocyclization of bilirubin in the presence of human serum albumin. Dependence of quantum yield on excitation wavelength. Photochem Photobiol 50:305-319

10. Als H 1998 Developmental care in the newborn intensive care unit. Curr Opin Pediatr 10:138-142

11. Glass P, Avery GB, Subramanian KN, Keys MP, Sostek AM, Friendly DS 1985 Effect of bright light in the hospital nursery on the incidence of retinopathy of prematurity. N Engl J Med 313:401-404

12. American Academy of Pediatrics Subcommittee on Hyperbilirubinemia 2004 Management of hyperbilirubinemia in the newborn infant 35 or more weeks of gestation. Pediatrics 114:297-316

13. Tan KL 1997 Efficacy of bidirectional fiberoptic phototherapy for neonatal hyperbilirubinemia. Pediatrics 99:E13

14. Garg AK, Prasad RS, Hifzi IA 1995 A controlled trial of high-intensity doublesurface phototherapy on a fluid bed versus conventional phototherapy in neonatal jaundice. Pediatrics 95:914-916

15. De Carvalho M, De Carvalho D, Trzmielina S, Lopes JM, Hansen TW 1999 Intensified phototherapy using daylight fluorescent lamps. Acta Paediatr 88:768-771

16. Agati G, Fusi F, Pratesi S, Galvan P, Donzelli GP 1998 Bilirubin photoisomerization products in serum and urine from a Crigler-Najjar type I patient treated by phototherapy. J Photochem Photobiol B 47:181-189

17. Djokomuljanto S, Quah BS, Surini Y, Noraida R, Ismail NZ, Hansen TW, Van Rostenberghe H 2006 Efficacy of phototherapy for neonatal jaundice is increased by the use of low-cost white reflecting curtains. Arch Dis Child Fetal Neonatal Ed 91:F439-F442

18. Sivanandan S, Chawla D, Misra S, Agarwal R, Deorari AK 2009 Effect of sling application on efficacy of phototherapy in healthy term neonates with nonhemolytic jaundice: a randomized conrolled trial. Indian Pediatr 46:23-28

19. Hansen TW 1997 Acute management of extreme neonatal jaundice-the potential benefits of intensified phototherapy and interruption of enterohepatic bilirubin circulation. Acta Paediatr 86:843-846

20. Harris MC, Bernbaum JC, Polin JR, Zimmerman R, Polin RA 2001 Developmental follow-up of breastfed term and near-term infants with marked hyperbilirubinemia. Pediatrics 107:1075-1080

21. Hansen TW, Nietsch L, Norman E, Bjerre JV, Hascoet JM, Mreihil K, Ebbesen F 2009 Apparent reversibility of acute intermediate phase bilirubin encephalopathy. Acta Paediatr 98:1689-1694

22. Walmsley D, Alzaharani K, Coke WJ, Ganghi R 2009 Total knee arthroplasty and Crigler-Najjar syndrome: a case report. Knee, Sep 12. [Epub ahead of print]

23. McDonagh AF, Lightner DA 1985 Like a shrivelled blood orange. Bilirubin, jaundice and phototherapy. Pediatrics 75:443-455 